\title{
Single-Antenna Doppler Radars Using Self and Mutual Injection Locking for Vital Sign Detection With Random Body Movement Cancellation
}

\author{
Fu-Kang Wang, Student Member, IEEE, Tzyy-Sheng Horng, Senior Member, IEEE, \\ Kang-Chun Peng, Member, IEEE, Je-Kuan Jau, Jian-Yu Li, Student Member, IEEE, and \\ Cheng-Chung Chen
}

\begin{abstract}
This work presents a single-antenna self-injection-locked (SIL) radar to reduce the hardware complexity of continuous-wave $(\mathrm{CW})$ Doppler systems. The theory provides a basis for determining the signal-to-noise spectral density ratio (SNDR) with the effects of clutter. Experimental results agree closely with the theoretical predictions, showing that the clutter does not affect the optimal SNR performance in an SIL radar. A single-antenna SIL radar array is designed to detect vital signs with random body movement cancellation. To this end, a subject is placed between two single-antenna SIL radars to measure the rates of respiration and heartbeat using Doppler shift, and the effects of random movement of the subject are cancelled by wireless mutual injection locking (MIL) of the two radars. In an experiment, a prototype of such a two-radar array with a spacing of $2 \mathrm{~m}$ was implemented at $2.4 \mathrm{GHz}$, providing accurate and reliable cardiopulmonary monitoring of a subject who jogged on a treadmill with random body motion of many centimeters.
\end{abstract}

Index Terms-Clutter effects, continuous-wave (CW) radar, Doppler radar, injection locking, random body movement cancellation, vital sign detection.

\section{INTRODUCTION}

C ONTINUOUS-WAVE (CW) Doppler radars have been developed for accurate and reliable cardiopulmonary monitoring [1]-[6]. They offer more flexibility in the monitoring sites on the patient than other methods do and thus makes the patient less uncomfortable. Several technical difficulties in the use of such radars have been overcome. For example, the null detection points at every odd multiple of one-eighth

Manuscript received July 06, 2011; revised September 26, 2011; accepted September 27, 2011. Date of publication November 16, 2011; date of current version December 14, 2011. This work was supported in part by the National Science Council, Taiwan, under Grant 97-2221-E-110-034-MY3, Grant 97-2221-E-110-035-MY3, Grant 100-2221-E-110-082-MY3, and Grant 99-2622-E-110-003-CC1, and by the Department of Industrial Technology, Taiwan, under Grant 99-EC-17-A-01-S1-104. This paper is an expanded paper from the IEEE international Microwave Symposium, Baltimore, MD, June 5-10, 2011.

F.-K. Wang and T.-S. Horng are with the Department of Electrical Engineering, National Sun Yat-Sen University, Kaohsiung 804, Taiwan (e-mail: d983010020@student.nsysu.edu.tw; jason@ee.nsysu.edu.tw).

K.-C. Peng is with Department of Computer and Communication Engineering, National Kaohsiung First University of Science and Technology, Kaohsiung 811, Taiwan (e-mail: peterpkg@ccms.nkfust.edu.tw).

J.-K. Jau, J.-Y. Li, and C.-C. Chen are with the Information and Communication Research Laboratory, ITRI, Hsinchu 310, Taiwan (e-mail: jekuan@itri. org.tw).

Color versions of one or more of the figures in this paper are available online at http://ieeexplore.ieee.org.

Digital Object Identifier 10.1109/TMTT.2011.2171712 wavelength from the radar to the subject can be eliminated using a quadrature architecture [1]. However, the quadrature imbalance often introduces a residual phase error, which reduces the accuracy of detection [2]. Since a CW Doppler radar with a higher operating frequency is more sensitive to detect small movements of the chest wall, millimeter-wave circuits are utilized to improve sensitivity [3]. However, designing robust millimeter-wave radars with low power consumption and low cost is still challenging. In our previous work [4], a two-antenna self-injection-locked (SIL) radar was proposed to achieve a high signal-to-noise spectral density ratio (SNR) in the demodulator output and to increase the sensing distance by a factor of four by doubling the operating frequency. Additionally, the null detection points and cofrequency interference can be eliminated using a swept frequency in the oscillator.

Recently, arctangent demodulation [5] and complex signal demodulation [6] are two popular methods for combining I/Q outputs into a single channel signal to improve detection accuracy. However, the clutter signals that result from antenna coupling and stationary object reflection degrade the detection performance. Thus, much effort has been made to solve this problem [7]-[10]. A possible approach uses a low-corner-frequency high-pass filter to remove concurrent dc offset and to preserve desired information [7]. In one approach [8], a generator outputs a signal with the same-amplitude but anti-phase to the received clutter signal to cancel the clutter effect at the RF frontend. Another work [9] presented a phase-diversity Doppler radar with minimized dc clutter noise. It included three antennas-one for transmitting and the other two for receiving, separated by half of a wavelength. The center estimation algorithm with dc cancellation [10] has been proposed to resolve this issue. However, these approaches [7]-[10] generally increase system complexity, cost, and power consumption.

Another great challenge that remains to be resolved is the influence of random body movement on the vital sign detection, because filtering the resultant Doppler shift out of the radar detection output is difficult. Only a few studies have addressed this issue. In one study [11], an empirical mode-decomposition method was adopted to remove the effects of body movement. However, it is effective only when the motion can be well represented as a set of intrinsic mode functions. Several methods [6], [12], [13] use two radars to cancel out the Doppler shift that is caused by random body motion. However, these methods rely on the use of different operating frequencies or orthogonal antenna polarization for enhancing isolation between the two radars, and 


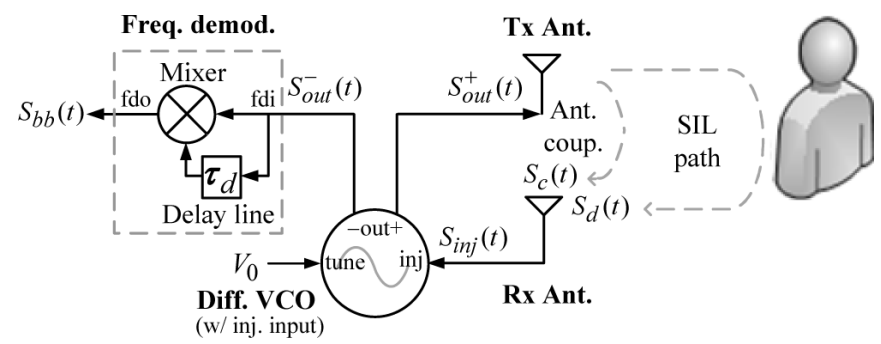

Fig. 1. Previously proposed two-antenna SIL radar architecture for vital sign detection.

the collection of baseband data from the two radars to cancel the body motion signal. Accordingly, the system complexity is also increased, leading to an excessive cost and power dissipation. Furthermore, the cancelled motion range is usually quite limited because random body movement and dc-clutter noise easily saturate the high-gain baseband circuit for amplifying vital-sign signals.

In this research, the single-antenna SIL radar is proposed to detect vital signs. Unlike the conventional single-antenna radar, which normally includes a circulator [14]-[16] or a coupler with high isolation [8] to separate transmitted and received signals, our proposed radar is based on self-injection locking to transmit and receive signals through the same antenna connected to one of the differential output terminals of an oscillator. Notably, there is no need to isolate the transmitted signal from the reflected signal in our proposed radar. Moreover, in the past, mutual injection locking (MIL) techniques were mainly used in phased arrays with beam-steering capabilities [17]-[19]. To the best of our knowledge, this research, for the first time, develops an MIL-based Doppler radar array for vital sign detection with random body movement cancellation. In an array structure, two SIL radars are synchronized by wireless MIL. Cancellation automatically occurs in the SIL oscillator stages under a specific MIL condition and thus does not need further processing of the baseband data from the two radars. Accordingly, almost no additional hardware beyond the two standalone radars is required. We have presented the self and mutual injection locking techniques for use in two-antenna Doppler radars to detect vital signs with random body movement cancellation in our preliminary publication [20]. This paper substantially expands the work in [20] to provide a single-antenna version of the system with careful analysis of the clutter effects. Experiments with the new proposed single-antenna SIL radars show very promising applications in fitness equipment with cardiopulmonary monitors.

\section{SIL RADAR Using A Single ANTENNA VERSUS TWO ANTENNAS}

As studied in our previous publications [4], [20], Fig. 1 shows the two-antenna SIL radar, which mainly contains a transmit (Tx) antenna, a receive ( $\mathrm{Rx}$ ) antenna, a differential voltage-controlled oscillator (VCO) using a push-pull configuration with an injection port, and a frequency demodulator composed of a passive mixer and a delay line. Both $\mathrm{Tx}$ and $\mathrm{Rx}$ antennas offer a high gain of more than $12 \mathrm{dBi}$ in the $2.4-\mathrm{GHz}$ ISM band. The VCO delivers a differential output power of about approximately $0 \mathrm{dBm}$ at a center frequency of $2.4 \mathrm{GHz}$ with a tuning

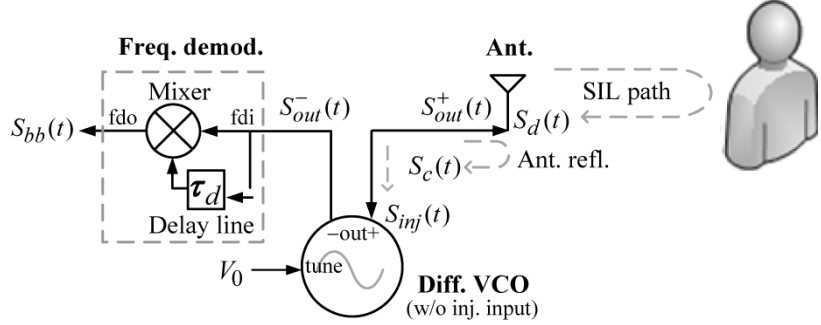

Fig. 2. New proposed single-antenna SIL radar architecture for vital-sign detection.

range of up to $500 \mathrm{MHz}$. The mixer has a conversion loss of about $7.5 \mathrm{~dB}$, and the delay line has a time delay of $22.5 \mathrm{~ns}$. The radar transmits the VCO's positive differential output signal $S_{\text {out }}^{+}(t)$ via one antenna and receives the reflected signal with Doppler phase modulation via another antenna. The received signal is then fed into the injection port of the VCO to form an SIL VCO. The clutter signal $S_{c}(t)$ due to coupling between Tx and Rx antennas can be very effectively reduced by properly choosing the beam width and direction of the two antennas. The frequency demodulator then extracts the vital-sign information from the VCO's negative differential output signal $S_{\text {out }}^{-}(t)$ with high SNDR.

In contrast, Fig. 2 shows the new proposed single-antenna SIL radar with the same components as in Fig. 1, except for the removal of the Rx injection input and antenna and the VCO output buffer. The radar uses the same antenna to transmit the CW output signal $S_{\text {out }}^{+}(t)$ toward the subject and to receive the reflected Doppler signal $S_{d}(t)$ without the need to isolate both signals. The received Doppler signal is injected into the differential VCO via the positive differential output terminal to establish an SIL loop. The clutter reflected from the antenna $S_{c}(t)$ actually has no influence on the optimal SNR performance of the SIL radar, which will be further validated in the following subsections.

\section{A. Experimental Setups}

Fig. 3(a) shows the setup for measuring the SNDR gain of the two-antenna SIL radar in Fig. 1. The circulator is used to isolate the output port from the injection port for the two-antenna SIL radar. The output signal $S_{\text {out }}^{+}(t)$ is divided into two signals. One is phase-modulated by a sinusoidal waveform of frequency $\omega_{m}$, phase-shifted, and attenuated to represent a Doppler signal $S_{d}(t)$; the other signal is phase-shifted, attenuated, but unmodulated to represent a clutter signal $S_{c}(t)$. Assume that the combined transmission coefficient of the attenuator and phase shifter is $A_{1} e^{i \phi_{1}}$ and $A_{2} e^{i \phi_{2}}$ in the path of the clutter signal $S_{c}(t)$ and the Doppler signal $S_{d}(t)$, respectively, while the splitter, combiner, and circulator are ideally lossless with zero time delay. After power combination, the injection signal $S_{\text {inj }}(t)$ is fed back via the circulator to establish the SIL loop and is given by

$$
\begin{aligned}
S_{\text {inj }}(t)= & S_{c}(t)+S_{d}(t) \\
= & \frac{A_{1}}{\sqrt{2}} e^{i \phi_{1}} S_{\text {out }}^{+}(t)+\frac{A_{2}}{\sqrt{2}} \\
& \times e^{i \phi_{2}} S_{\text {out }}^{+}(t) * h(t), \text { for Fig. 3(a) }
\end{aligned}
$$




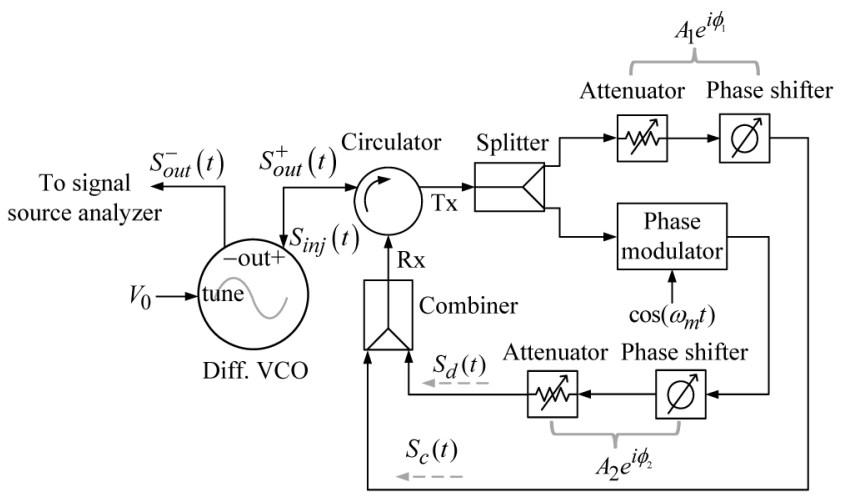

(a)

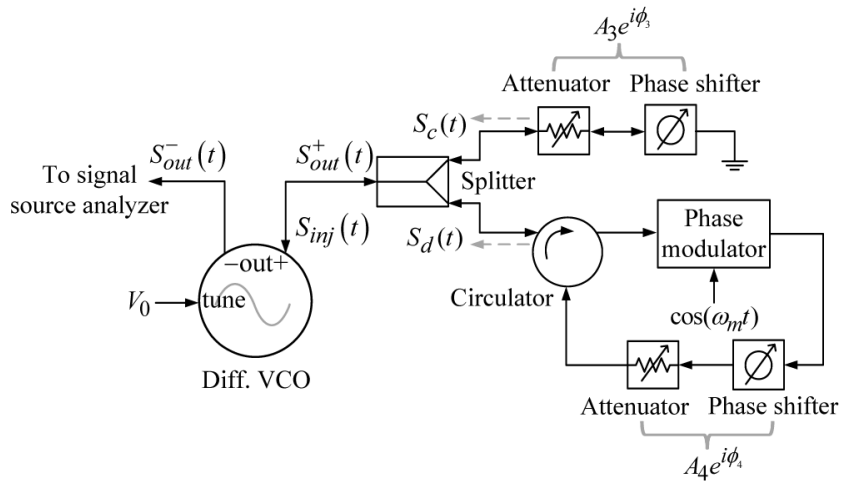

(b)

Fig. 3. Experimental setups for measuring the SNR gain of different SIL radar architectures. (a) Experiment 1 for two-antenna SIL radar architecture. (b) Experiment 2 for single-antenna SIL radar architecture.

where $h(t)$ is the impulse response of the phase modulator.

Fig. 3(b) shows the SNDR measurement setup for the singleantenna SIL radar in Fig. 2 using the same differential VCO, the same phase modulator, and the same ideal splitter and circulator as in Fig. 3(a). The output signal $S_{\text {out }}^{+}(t)$ splits into two signals and combines them after each signal has passed through a different path. As shown in Fig. 3(b), one signal leaves and returns via the path with a phase shifter, an attenuator, and a short circuit at the end to represent a clutter signal $S_{c}(t)$; the other signal is phase modulated, phase-shifted, attenuated and then fed back via a circulator to represent a Doppler signal $S_{d}(t)$. Let us also assume that the combined transmission coefficient of the attenuator and phase shifter in the clutter and Doppler signal path is $A_{3} e^{i \phi_{3}}$ and $A_{4} e^{i \phi_{4}}$, respectively. In this architecture, the VCO uses the same port to transmit the output signal $S_{\text {out }}^{+}(t)$ and receive the injection signal $S_{\mathrm{inj}}(t)$, where $S_{\mathrm{inj}}(t)$ is given by

$$
\begin{aligned}
S_{\mathrm{inj}}(t)= & S_{c}(t)+S_{d}(t) \\
= & -\frac{A_{3}^{2}}{\sqrt{2}} e^{i 2 \phi_{3}} S_{\text {out }}^{+}(t)+\frac{A_{4}}{\sqrt{2}} \\
& \times e^{i \phi_{4}} S_{\text {out }}^{+}(t) * h(t), \quad \text { for Fig. 3(b). }
\end{aligned}
$$

Notably, the attenuator and the phase shifter in Fig. 3(a) and (b) can both be varied to adjust the injection amplitude and phase shift. The signal in the other differential output terminal $S_{\text {out }}^{-}(t)$ is sent to a signal source analyzer to measure its power spectral density (PSD). A constant voltage $V_{0}$ is applied to determine the oscillation frequency $\omega_{\text {osc }}$. From
(1) and (2), the two architectures generally yield the same performance when the following four conditions are satisfied: $A_{1}=A_{3}^{2}, \phi_{1}=2 \phi_{3}-\pi, A_{2}=A_{4}$, and $\phi_{2}=\phi_{4}$.

\section{B. System Model With Clutter Effects}

As shown in Figs. 1 and 2, an RF CW signal is generated by the VCO and emitted toward the subject. The time-varying position of the chest wall, caused by cardiopulmonary activities, modulates the phase of the transmitted signal. However, clutter from antenna coupling, as shown in Fig. 1, and antenna reflection, as shown in Fig. 2, occur in the testing environment. The clutter signal has the same frequency as the transmitted signal but without the phase modulation that is determined by vitalsign information. The radar then receives the Doppler signal and clutter signal and injects them into the VCO to cause the VCO to enter an SIL state.

Therefore, the injection signals have two components: the Doppler signal $S_{d}(t)$ caused by the cardiopulmonary activities of subject, which has a constant amplitude $E_{\mathrm{inj}, d}$ and an instantaneous frequency $\omega_{\mathrm{inj}, d}(t)$, and the antenna clutter signal $S_{c}(t)$ with constant amplitude $E_{\mathrm{inj}, c}$ and instantaneous frequency $\omega_{\mathrm{inj}, c}(t)$. Based on Adler's analysis [21], when $S_{\mathrm{inj}}(t)$ enters the VCO, the output signal can be regarded as a vector that rotates clockwise with a beat frequency $d \alpha(t) / d t$ with respect to $S_{\mathrm{inj}}(t)$, where $\alpha(t)$ is the phase difference from $S_{\mathrm{inj}}(t)$ to $S_{\mathrm{osc}}(t)$. Hence, the instantaneous VCO output frequency $\omega_{\text {out }}(t)$ is given by

$$
\omega_{\text {out }}(t)=\omega_{\text {inj }}(t)+\frac{d \alpha(t)}{d t}
$$

where

$$
\alpha(t)=\alpha_{c}(t)+\alpha_{d}(t) .
$$

$\alpha_{c}(t)$ and $\alpha_{d}(t)$ represent the phase differences from $S_{c}(t)$ and $S_{d}(t)$ to $S_{\text {osc }}(t)$, respectively. From Figs. 1 and 2, the clutter signal arising from antenna coupling or antenna reflection clearly has a shorter propagation delay than the Doppler signal that is reflected from a subject. Thus, the Adler's equation [21] with respect to the clutter signal and the Doppler signal can be written, respectively, as

$$
\begin{aligned}
\frac{d \alpha_{c}}{d t} & =\omega_{\mathrm{osc}}-\omega_{\mathrm{inj}, c}(t)-\omega_{\mathrm{LR}, c} \sin \alpha_{c}(t) \\
\frac{d \alpha_{d}}{d t} & =\omega_{\mathrm{inj}, c}(t)-\omega_{\mathrm{out}}(t)-\omega_{\mathrm{LR}, d} \sin \alpha_{d}(t) .
\end{aligned}
$$

In (5) and (6), the locking ranges for the two signals are respectively given by

$$
\begin{aligned}
& \omega_{\mathrm{LR}, c}=\frac{\omega_{\mathrm{osc}}}{2 Q_{\mathrm{tank}}} \frac{E_{\mathrm{inj}, c}}{E_{\mathrm{osc}}} \\
& \omega_{\mathrm{LR}, d}=\frac{\omega_{\mathrm{inj}, c}}{2 Q_{\mathrm{tank}}} \frac{E_{\mathrm{inj}, d}}{E_{\mathrm{osc}}}
\end{aligned}
$$

where $Q_{\operatorname{tank}}$ is a quality factor of the VCO's tank circuit. Formulas (4)-(6) yield

$$
\frac{d \alpha(t)}{d t}=\omega_{\text {osc }}-\omega_{\text {out }}(t)-\omega_{\mathrm{LR}, c} \sin \alpha_{c}(t)-\omega_{\mathrm{LR}, d} \sin \alpha_{d}(t) .
$$


When the SIL VCO is in the steady state, the time-varying variables in (9) approach constant values. Therefore, (9) equals 0 , yielding

$$
\omega_{\mathrm{out}}=\omega_{\mathrm{osc}}-\omega_{\mathrm{LR}, c} \sin \phi_{c}-\omega_{\mathrm{LR}, d} \sin \phi_{d}
$$

where $\omega_{\text {out }}, \phi_{c}$, and $\phi_{d}$ are the steady-state values of $\omega_{\text {out }}(t), \alpha_{c}(t)$, and $\alpha_{d}(t)$, respectively. Notably, under the SIL condition, $\phi_{c}$ and $\phi_{d}$ are equal to the propagation phase delay of the clutter signal and the Doppler signal, respectively, and are given by

$$
\begin{aligned}
& \phi_{c}=2 \omega_{\mathrm{out}} \tau_{c} \\
& \phi_{d}=2 \omega_{\mathrm{out}} \tau_{d}
\end{aligned}
$$

where $2 \tau_{c}$ and $2 \tau_{d}$ represent the round-trip path delay of the clutter signal and the Doppler signal, respectively.

By referring to the frequency-domain model established in [4] for an SIL VCO, the VCO output phase shift $\phi_{\text {out }}(s)$ can be determined as a combination of the Doppler phase modulation $\phi_{v}(s)$ and the VCO output phase noise $\phi_{n}(s)$, that is,

$$
\phi_{\text {out }}(s)=H_{m}(s) \cdot \phi_{v}(s)+H_{o}(s) \cdot \phi_{n}(s)
$$

where, for $s \tau_{d} \ll 1$

$$
H_{m}(s) \approx \frac{\omega_{\mathrm{LR}, d} \cos \phi_{d}}{s\left(1+\omega_{\mathrm{LR}, d} \cos \phi_{d} \cdot 2 \tau_{d}\right)}
$$

is a transfer function of integration with respect to $\phi_{v}(s)$ and

$$
H_{o}(s) \approx \frac{1}{1+\omega_{\mathrm{LR}, d} \cos \phi_{d} \cdot 2 \tau_{d}}
$$

is a transfer function of suppression with respect to $\phi_{n}(s)$. To extract the phase-modulated signal, a frequency demodulator that is based on delay-line discrimination is subsequently applied to process the VCO output signal. For a time delay $\tau_{f}$ in the delay-line discrimination process, the baseband phase detection output $\phi_{b b}(s)$ can be expressed as

$$
\begin{aligned}
\phi_{b b}(s)= & \phi_{\mathrm{out}}(s) \cdot s \tau_{f} \\
= & \frac{\omega_{\mathrm{LR}, d} \cos \phi_{d} \cdot \tau_{f}}{1+2 \omega_{\mathrm{LR}, d} \cos \phi_{d} \cdot \tau_{d}} \cdot \phi_{v}(s) \\
& +\frac{s \tau_{f}}{1+2 \omega_{\mathrm{LR}, d} \cos \phi_{d} \cdot \tau_{d}} \cdot \phi_{n}(s) .
\end{aligned}
$$

From (16), the SIL radar architecture functions as a firstorder delta-sigma modulator for noise shaping to enhance the SNDR of the phase-modulated signal. The SIL mechanism acts like an integrator to reinforce the low-frequency components of the phase-modulated signal and like an attenuator to suppress the phase noise. In contrast, frequency demodulation involves a differential operation to restore the phase-modulation signal without distortion and, in the meantime, push the phase noise out of the modulation bandwidth to higher frequencies. The SNDR gain is defined as the ratio of the SNDR after to that before the delta-sigma modulation process, and it is of the form

$$
G_{\mathrm{SNR}}=\frac{\left|H_{m}(s)\right|^{2}}{\left|H_{o}(s)\right|^{2}}=\left|\frac{\omega_{\mathrm{LR}, d} \cos \phi_{d}}{s}\right|^{2} .
$$

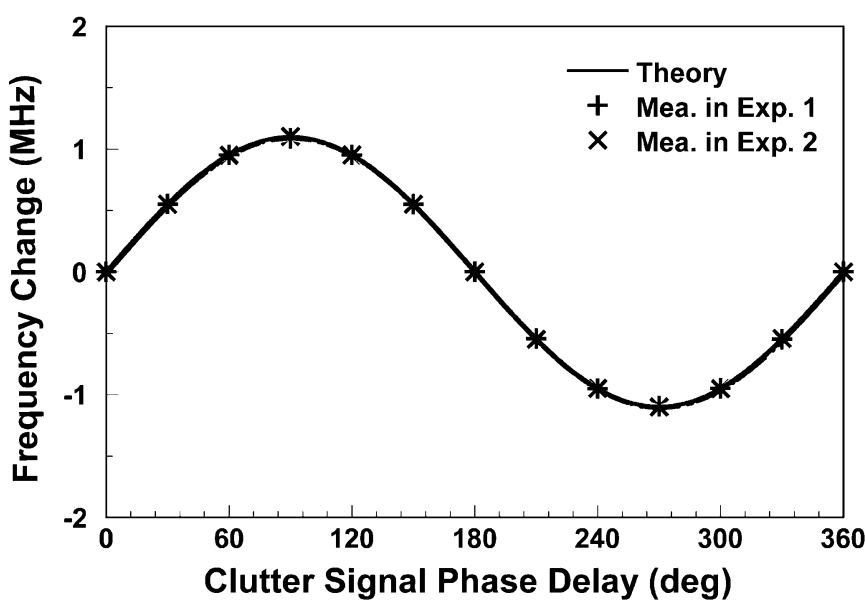

Fig. 4. Oscillation frequency change $\Delta \omega$ versus clutter signal phase delay $\phi_{c}$.

\section{SNDR Gain Experiments}

Next, measurements that verify the developed theory are presented. Fig. 3(a) and (b) shows the experimental setups for twoantenna and single-antenna SIL radars, which have the same SNDR gain under the SIL condition if the attenuation and phase shift of each path are properly tuned.

First, the Doppler signal paths in the two experimental setups are terminated by matched loads to measure the frequency change from $\omega_{\text {osc }}$ to $\omega_{\text {out }}$ because of the clutter effects. For $E_{\mathrm{inj}, d} \approx 0$, this frequency change can be obtained from (10) as

$$
\Delta \omega=\omega_{\mathrm{osc}}-\omega_{\mathrm{out}} \approx \omega_{\mathrm{LR}, c} \sin \phi_{c}
$$

Fig. 4 compares the theoretical and measured results versus the clutter signal phase delay $\phi_{c}$. In this experiment, the $\mathrm{VCO}$ with a tank quality factor of around 34 initially oscillates at $2.480 \mathrm{GHz}$. The clutter to oscillation signal amplitude ratio $E_{\text {inj }, c} / E_{\text {osc }}$ is set as 0.03 . The calculated corresponding locking range $\omega_{\mathrm{LR}, c}$ is about $2 \pi \times 1.1 \mathrm{MHz}$. In Fig. 4, the solid line represents the calculated frequency change using (18). The circle and diamond symbols respectively represent the measured results using two experimental setups. These three sets of results agree very closely with each other. The maximum frequency change is $\pm 1.1 \mathrm{MHz}$ as $\phi_{c}$ is equal to $90^{\circ}$ or $270^{\circ}$.

Fig. 5 shows the clutter effect on the detection of a Doppler signal with an amplitude ratio $E_{\mathrm{inj}, d} / E_{\text {osc }}$ set equal to 0.007 . The black line represents the calculated SNDR gain versus the Doppler signal phase delay $\phi_{d}$ when the clutter signal is terminated by a matched load rather than injected into the VCO in the two experimental setups. Considering the clutter signal that causes the maximum frequency change of $1.1 \mathrm{MHz}$ at a clutter signal phase delay of $90^{\circ}$, the clutter-affected SNR gain is plotted according to (17), as shown by the gray line in Fig. 5. Notably, the clutter signal frequency $\omega_{\text {inj,c }}$ required for evaluating the locking range $\omega_{\mathrm{LR}, d}$ in (17) is almost equal to $\omega_{\text {out }}$ in the steady state. The plus signs in black, cross signs in black, plus signs in gray, and cross signs in gray represent the measurements made using experimental setup 1 without clutter, experimental setup 2 without clutter, experimental setup 1 with clutter, and experimental setup 2 with clutter, respectively. Again, there 


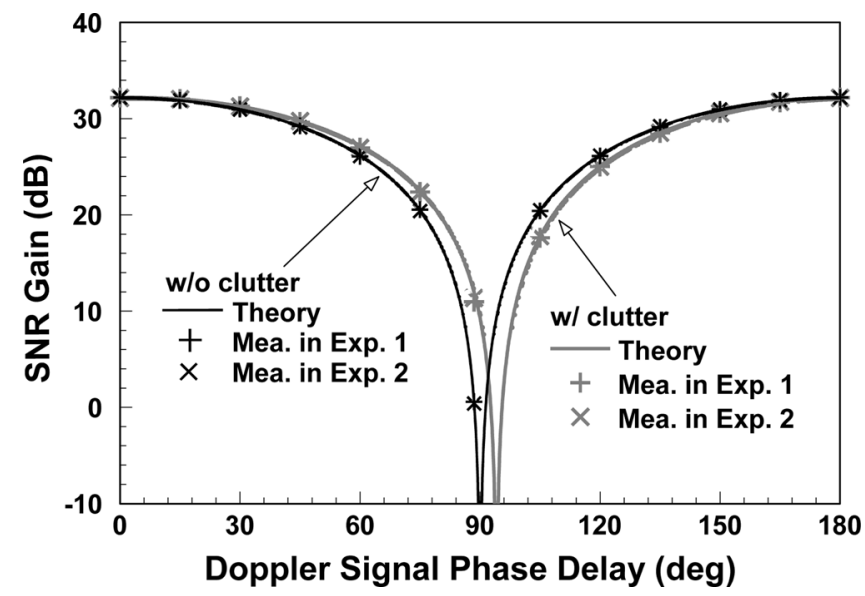

Fig. 5. SNDR gain versus Doppler signal phase delay $\phi_{d}$.

is excellent agreement between the measurements and theoretical predictions. Clearly, Fig. 5 shows that the clutter effect is equivalent to an additional shift of $\phi_{d}$, which does not affect the maximum SNDR gain. Restated, the clutter signal has an impact on the steady-state VCO output frequency and then causes a change in the Doppler signal phase delay $\phi_{d}$ and subsequently in the SNDR gain $G_{\mathrm{SNR}}$. However, the single-antenna SIL radar with considerable clutter can achieve the same maximum SNDR gain as the two-antenna SIL radar with minimized clutter if its operating frequency or the position of the subject is properly adjusted.

\section{Vital-Sign Sensing Experiments}

In the following experiments for vital sign detection, an adult who is breathing normally is seated one meter from the radar, whose initial output frequency is at $2.4 \mathrm{GHz}$. Figs. 6(a) and 6(b) show the experimental setups for the two radar architectures, with no object within sensing distance in front of the radar. In Fig. $6(\mathrm{c})$, a $60 \times 60 \mathrm{~cm}^{2}$ metal plate is placed near the subject to cause another stationary clutter in the radar reception. In Figs. 6(a)-(c), the one-meter distance between the subject and the SIL radar is eight wavelengths long at $2.4 \mathrm{GHz}$, which indicates that the subject is at the optimal detection point for maximum SNR gain because $\phi_{d}$ is $32 \pi$ in evaluating (17). In contrast, Fig. 6(d) illustrates another single-antenna SIL radar experiemnt to detect the subject's vital signs at a null detection point with a sensing distance of $1.016 \mathrm{~m}$ or a $\phi_{d}$ of $32.5 \pi$. In the above experiments, the transmission coefficient between $\mathrm{Tx}$ and Rx antennas in Fig. 6(a) is around 0.03. As for the single-antenna SIL radars in Figs. 6(b)-(d), the antenna reflection coefficient is around 0.2. The radar detection output signals were processed by a digital filter with the passband $0.1-10 \mathrm{~Hz}$.

Fig. 7 plots the detected vital-sign waveforms in the four experiments of Fig. 6. The root-mean-square (rms) values of the waveforms in Fig. 6(a)-(c) are 0.825, 0.823, and $0.822 \mathrm{~V}$, respectively, revealing that the three kinds of clutter have very little influence on the detection outcome when the subject is at the optimal detection point. In Fig. 6(d), the gray line exhibits an output vital-sign waveform with vanishing amplitude because the subject was at a null detection point when the SIL radar operated at a single frequency of $2.4 \mathrm{GHz}$. However, by instead performing a frequency sweep in the ISM band from 2.4 to $2.484 \mathrm{GHz}$, the radar provided

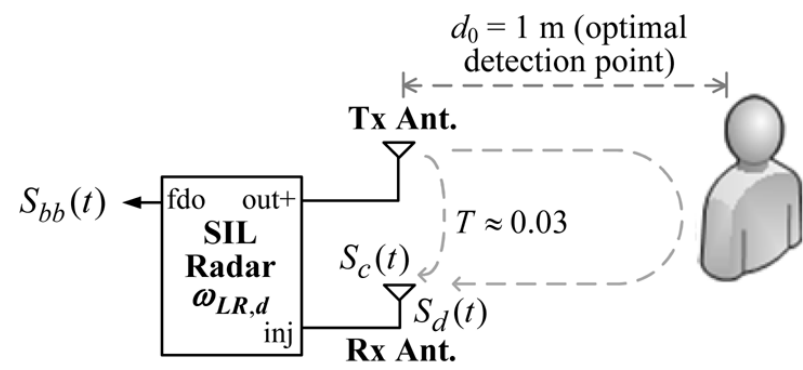

(a)

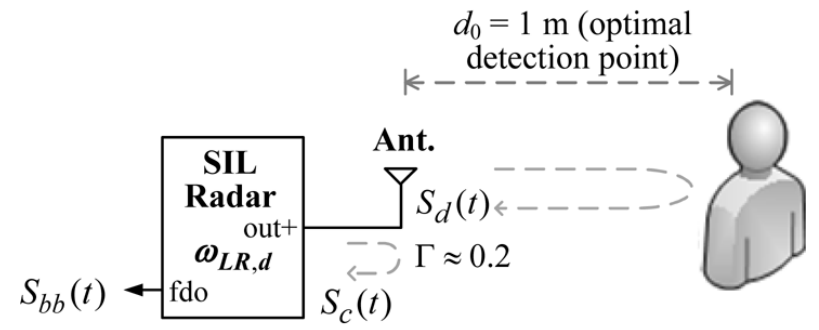

(b)

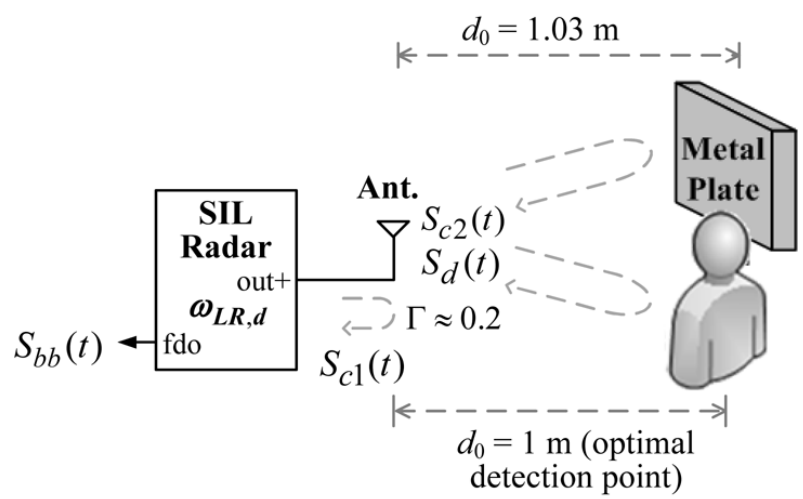

(c)

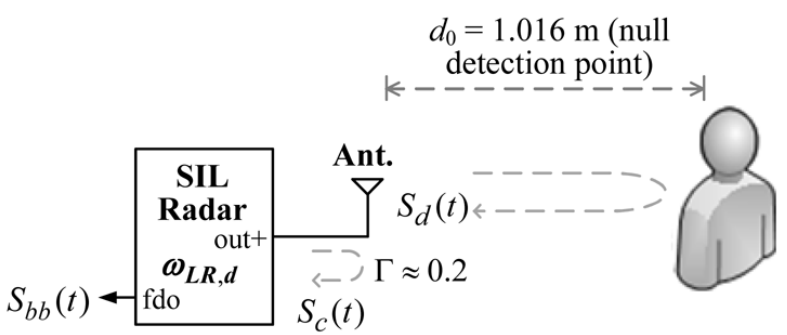

(d)

Fig. 6. Experimental setups for using SIL radars to detect vital signs of a subject. (a) Two-antenna SIL radar with the effect of antenna coupling clutter. (b) Single-antenna SIL radar with the effect of antenna reflection clutter. (c) Singleantenna SIL radar with the combined effect of antenna reflection clutter and stationary object clutter. (d) Single-antenna SIL radar with the problem of null detection point.

an output waveform with sufficient amplitude for vital sign detection, as plotted by the black line.

Fig. 8 shows the Fourier-transformed output spectra. In Fig. 8(a)-(c), the peak voltages associated with respiration are $1.074,1.061$, and $1.042 \mathrm{~V}$, respectively. Those associated with heartbeat are $0.189,0.183$, and $0.169 \mathrm{~V}$, respectively. All three outcomes provide sufficient amplitude to identify vital-sign information: a breathing rate of 18 beats/min and a heartbeat rate of 78 beats/min. Based on the above experiments 


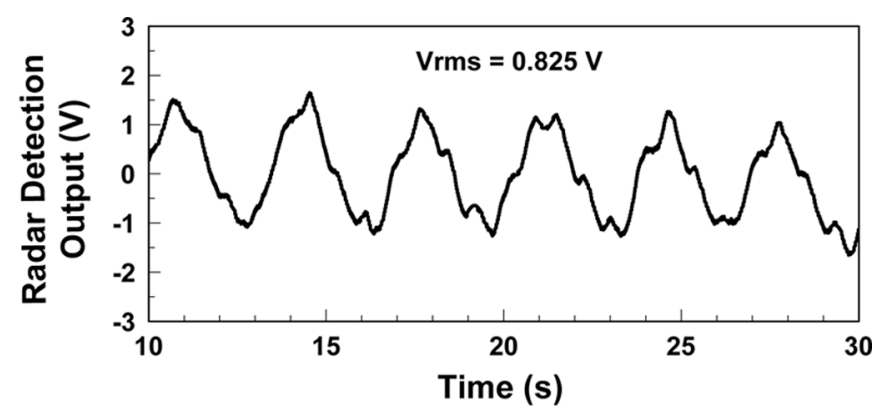

(a)

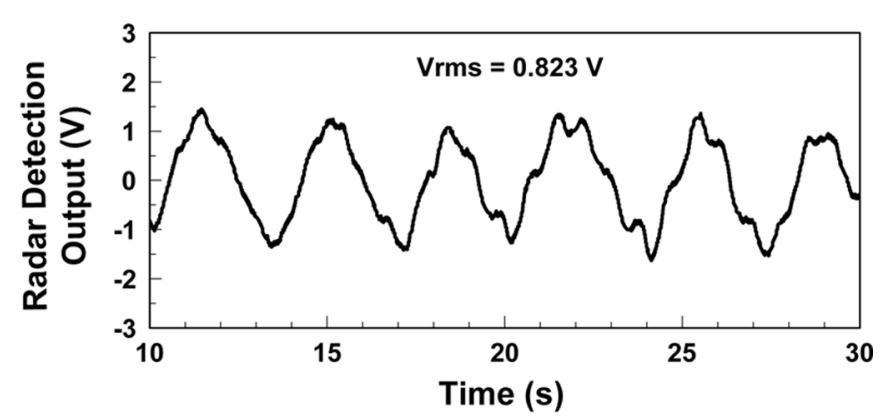

(b)

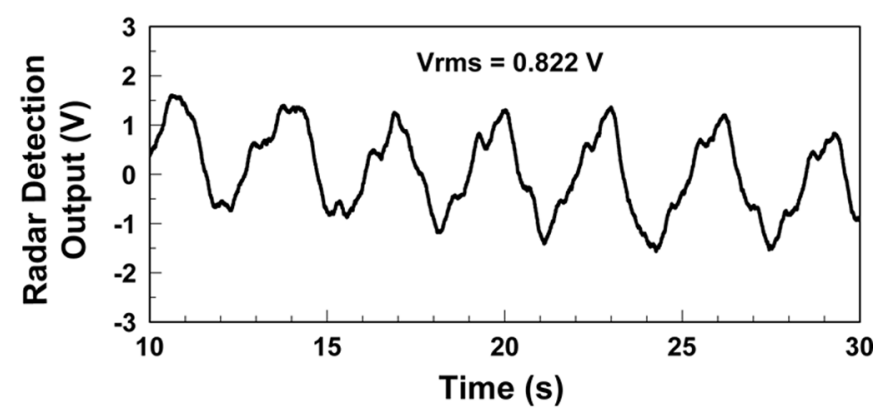

(c)

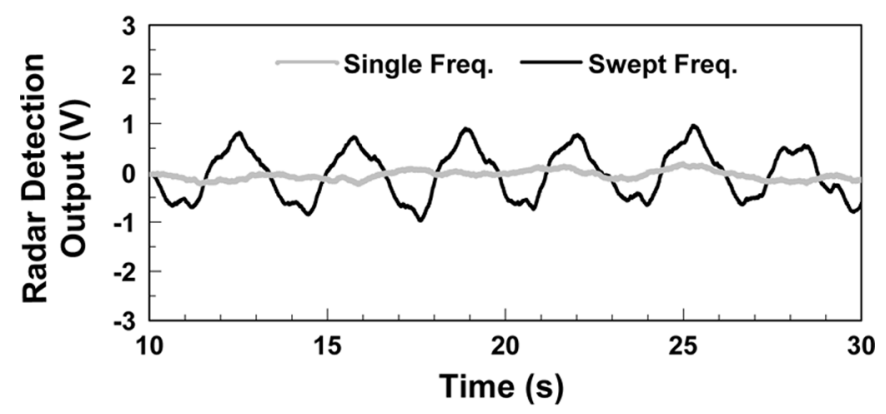

(d)

Fig. 7. Detected vital-sign waveforms for different experimental setups in Fig. 6. (a) Setup of Fig. 6(a). (b) Setup of Fig. 6(b). (c) Setup of Fig. 6(c). (d) Setup of Fig. 6(d).

and theory, the SIL radar has strong resistance to any kinds of stationary clutter. Therefore, the two-antenna SIL radar, which aims at mitigating the antenna clutter, can be replaced by the single-antenna SIL radar, since the antenna clutter actually has negligible impact on the SIL radars for Doppler detection. The obvious advantages include low system complexity, low cost, low power consumption, and high SNDR performance. Fig. 8(d) compares the vital-sign spectra from the single-antenna SIL radar output between the detected results shown
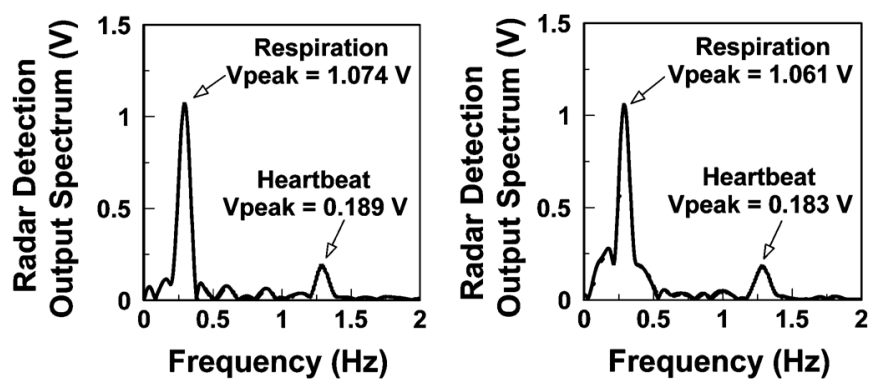

(a)

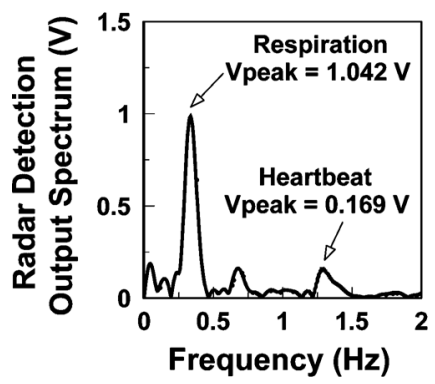

(c)

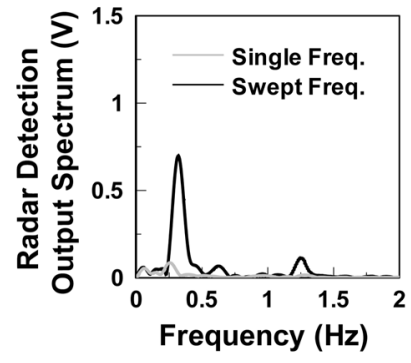

(d)
Fig. 8. Detected vital-sign spectra for different experimental setups in Fig. 6. (a) Setup of Fig. 6(a). (b) Setup of Fig. 6(b). (c) Setup of Fig. 6(c). (d) Setup of Fig. 6(d).

by the gray line that were obtained at a null detection point with a single frequency and the improved results shown by the black line that were obtained using a swept frequency. Clearly, the use of swept frequency can effectively resolve the null detection point problem. A breathing rate of 19 beats/min and a heartbeat rate of 75 beats/min can be identified by the black line in Fig. 8(d).

\section{Two Mutually INJECTION-LOCKED SIL RADARS FOR RANDOM BODY MOVEMENT CANCELLATION}

Fig. 9 displays the proposed two-radar array for cancelling the effects of random body movement on the detection of vital signs. In Fig. 9, a subject is assumed to move back and forth randomly between two single-antenna SIL radars, one in front and one behind the subject, with an instantaneous displacement $x_{b}(t)$. The two radars, SIL radars 1 and 2, with different locking ranges $\omega_{\mathrm{LR} 1, d}$ and $\omega_{\mathrm{LR} 2, d}$ are mutually injection-locked through their antennas with the proper beam width and direction. Notably, in Fig. 9, the round-trip propagation delays from SIL radar 1 to the front of the subject and from SIL radar 2 to the back of the subject equal $2 \tau_{d 1}$ and $2 \tau_{d 2}$, respectively, and the propagation delay over a body thickness equals $\tau_{b}$. Therefore, the one-way propagation delay $\tau_{s}$ between the two radars can be regarded as $\tau_{d 1}+\tau_{d 2}+\tau_{b}$.

Fig. 10 shows the equivalent frequency-domain model for the proposed two mutually injection-locked SIL radars. Under the MIL condition, the VCO output phase signals in both radars are given by

$$
\begin{aligned}
& \phi_{s 1}(s)=\frac{\omega_{\mathrm{LR} 1, d}}{s+\omega_{\mathrm{LR} 1, d}} \phi_{s 2}(s) e^{-s \tau_{s}}+\frac{s}{s+\omega_{\mathrm{LR} 1, d}} \phi_{\mathrm{out} 1}(s) \\
& \phi_{s 2}(s)=\frac{\omega_{\mathrm{LR} 2, d}}{s+\omega_{\mathrm{LR} 2, d}} \phi_{s 1}(s) e^{-s \tau_{s}}+\frac{s}{s+\omega_{\mathrm{LR} 2, d}} \phi_{\mathrm{out} 2}(s)
\end{aligned}
$$




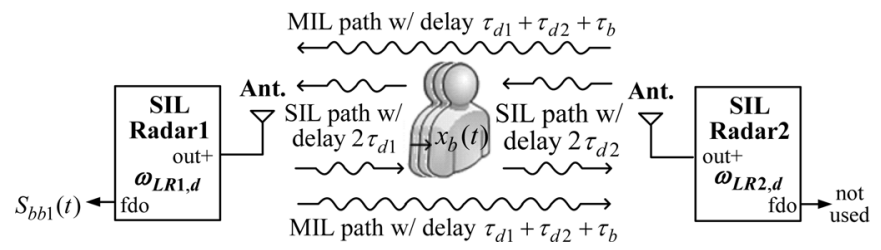

Fig. 9. Proposed two mutually injection-locked SIL radars for cancelling the effects of random body movement on vital-sign detection.

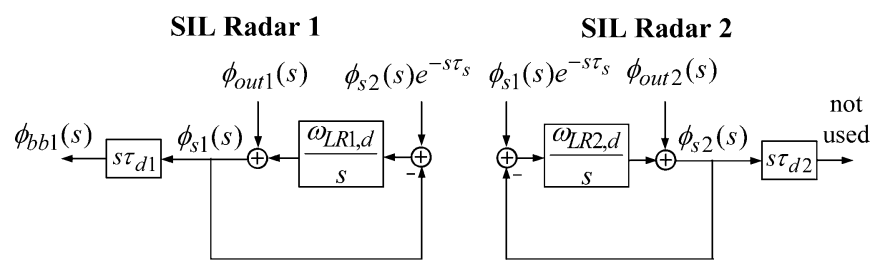

Fig. 10. Frequency-domain model of the proposed two mutually injection-locked SIL radars.

where

$$
\begin{aligned}
& \phi_{\text {out } 1}(s)=H_{m 1}(s) \cdot\left(\phi_{v}(s)-\phi_{b}(s)\right)+H_{o 1}(s) \cdot \phi_{n 1}(s) \\
& \phi_{\text {out } 2}(s)=H_{m 2}(s) \cdot\left(\phi_{v}(s)+\phi_{b}(s)\right)+H_{o 2}(s) \cdot \phi_{n 1}(s)
\end{aligned}
$$

are the VCO output phase shifts in both radars under the SIL condition. Notably, in (21) and (22), $\phi_{v}(s)$ and $\phi_{b}(s)$ represent the phase modulation due to vital signs and movement of the body, respectively. Since vital signs are associated with an inward-and-outward chest motion, whereas body movement is back and forth, the resultant phase modulation in the detection outputs of the SIL radars 1 and 2 are the difference between two individual Doppler shifts and the sum of the two individual Doppler shifts. Solving (19) and (20) for the VCO output phase shift $\phi_{s 1}(s)$ in SIL radar 1 under the MIL condition gives

$$
\begin{aligned}
& \phi_{s 1}(s) \\
& \quad=\frac{s \phi_{\mathrm{out} 1}(s)+\left(\omega_{\mathrm{LR} 1, d} \phi_{\mathrm{out} 2}(s) \cdot e^{-s \tau_{s}}+\omega_{\mathrm{LR} 2, d} \phi_{\mathrm{out} 1}(s)\right)}{s+\left(\omega_{\mathrm{LR} 1, d}+\omega_{\mathrm{LR} 2, d}\right)+\omega_{\mathrm{LR} 1, d} \omega_{\mathrm{LR} 2, d}\left(1-e^{-s \cdot 2 \tau_{s}}\right) / s} \\
& \approx \frac{\omega_{\mathrm{LR} 1, d} \phi_{\mathrm{out} 2}(s)+\omega_{\mathrm{LR} 2, d} \phi_{\mathrm{out} 1}(s)}{\omega_{\mathrm{LR} 1, d}+\omega_{\mathrm{LR} 2, d}+2 \omega_{\mathrm{LR} 1, d} \omega_{\mathrm{LR} 2, d} \tau_{s}}, \quad \text { for } s \tau_{s} \ll 1 .
\end{aligned}
$$

Substituting (21) and (22) into (23) yields

$$
\begin{aligned}
\phi_{s 1}(s) \approx & \frac{\omega_{\mathrm{LR} 1, d} H_{m 2}(s)+\omega_{\mathrm{LR} 2, d} H_{m 1}(s)}{\omega_{\mathrm{LR} 1, d}+\omega_{\mathrm{LR} 2, d}+2 \omega_{\mathrm{LR} 1, d} \omega_{\mathrm{LR} 2, d} \tau_{s}} \phi_{v}(s) \\
& +\frac{\omega_{\mathrm{LR} 1, d} H_{m 2}(s)-\omega_{\mathrm{LR} 2, d} H_{m 1}(s)}{\omega_{\mathrm{LR} 1, d}+\omega_{\mathrm{LR} 2, d}+2 \omega_{\mathrm{LR} 1, d} \omega_{\mathrm{LR} 2, d} \tau_{s}} \phi_{b}(s) \\
& +\frac{\omega_{\mathrm{LR} 1, d} H_{o 2}(s) \phi_{n 2}(s)+\omega_{\mathrm{LR} 2, d} H_{o 1}(s) \phi_{n 1}(s)}{\omega_{\mathrm{LR} 1, d}+\omega_{\mathrm{LR} 2, d}+2 \omega_{\mathrm{LR} 1, d} \omega_{\mathrm{LR} 2, d} \tau_{s}} .
\end{aligned}
$$

$\phi_{s 2}(s)$ can be derived similarly. However, it need not be because the baseband data from SIL radar 2 are not used. To cancel the effects of body movement, the second term on the right-hand side of (24) must vanish, yielding the cancellation condition

$$
\omega_{\mathrm{LR} 1, d} H_{m 2}(s)=\omega_{\mathrm{LR} 2, d} H_{m 1}(s) .
$$

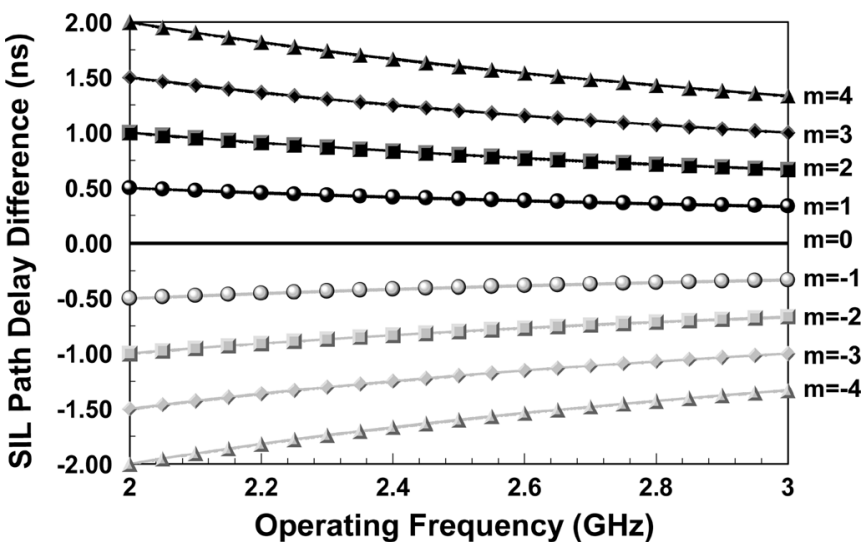

Fig. 11. SIL path delay difference versus radar's operating frequency under the condition of random body movement cancellation.

Further, substituting (14) into (25) gives

$$
\frac{\cos \phi_{d 2}}{1+2 \omega_{\mathrm{LR} 2, d} \cos \phi_{d 2} \cdot \tau_{d 2}}=\frac{\cos \phi_{d 1}}{1+2 \omega_{\mathrm{LR} 1, d} \cos \phi_{d 1} \cdot \tau_{d 1}}
$$

where $\phi_{d 1}$ and $\phi_{d 1}$ represent the Doppler signal phase delay for SIL radars 1 and 2, respectively. Since the two radars operate at the same frequency $\omega_{\text {out }}$ under the MIL condition, from (12), we have that $\phi_{d 1}=2 \omega_{\text {out }} \tau_{d 1}$ and $\phi_{d 2}=2 \omega_{\text {out }} \tau_{d 2}$. For $\omega_{\mathrm{LR} 1, d} \tau_{s} \ll 1$ and $\omega_{\mathrm{LR} 2, d} \tau_{s} \ll 1$,

$$
\Delta \tau_{d}=2\left(\tau_{d 1}-\tau_{d 2}\right)=\frac{2 m \pi}{\omega_{\text {out }}}, m \in N
$$

is the cancellation condition for random body movement. Fig. 11 explains the relation between the SIL path delay difference $\left(\Delta \tau_{d}\right)$ and the radar's operating frequency $\left(\omega_{\text {out }}\right)$ according to (27). It can be seen from the figure that, to achieve the cancellation condition, $\omega_{\text {out }}$ should be particularly chosen for a given $\Delta \tau_{d}$ unless $\Delta \tau_{d}=0$. Therefore, the cancellation of random body motion can be realized by varying the radar's operating frequency or the subject's offset position with respect to the center between two radars, and is approximately independent of the locking range of the individual radars. If none of the above two options are allowed in practical applications, an additional phase shifter can then be used to achieve the cancellation condition, as demonstrated in [20].

Under the cancellation condition, the baseband phase detection output in SIL radar 1 is derived as

$$
\begin{aligned}
\phi_{b b 1}(s) \approx & \frac{2 \omega_{\mathrm{LR} 1, d} \omega_{\mathrm{LR} 2, d} \tau_{f 1} \cos \phi_{d 1}}{\omega_{\mathrm{LR} 1, d}+\omega_{\mathrm{LR} 2, d}} \phi_{v}(s) \\
& +\frac{s \tau_{f 1} \cdot\left(\omega_{\mathrm{LR} 2, d} \phi_{n 1}(s)+\omega_{\mathrm{LR} 1, d} \phi_{n 2}(s)\right)}{\omega_{\mathrm{LR} 1, d}+\omega_{\mathrm{LR} 2, d}} .
\end{aligned}
$$

According to (28), the optimal SNR of the two-radar array detection output is obtained by satisfying

$$
\phi_{d 1}=\omega_{\text {out }} \cdot 2 \tau_{d 1}=2 n \pi, n \in N .
$$

Combining conditions (27) and (29) can determine $\omega_{\text {out }}$ and $\Delta \tau_{d}$ completely. 


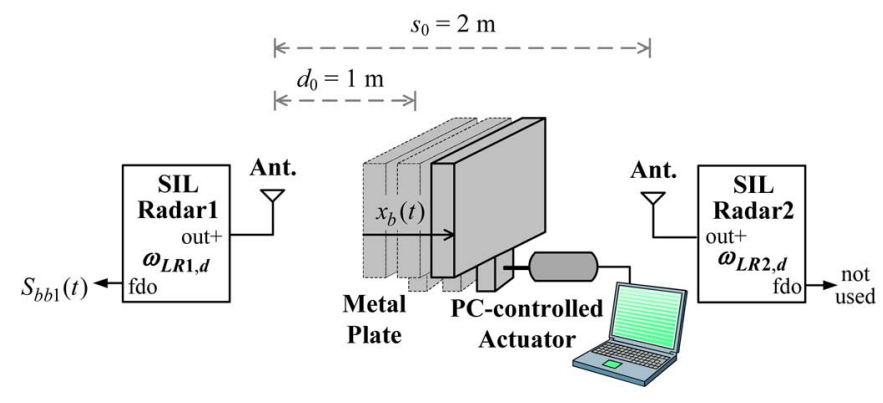

Fig. 12. Experiment setup for cancelling the Doppler shift caused by a backand-forth motion.

\section{EXPERIMENTAL RESULTS AND DISCUSSION}

Fig. 12 shows an experimental setup, with a metal plate placed at the center between the two single-antenna SIL radars, to verify the effectiveness of the proposed method for cancelling the effects of random body movement. Both radars are mutually injection-locked at the same frequency (approximately $2.4 \mathrm{GHz}$ ), and the distance between the two radars $s_{0}$ is $2 \mathrm{~m}$. A PC-controlled actuator is used to move the metal plate back and forth with an instantaneous displacement of

$$
x_{b}(t)=x_{b \max } \Lambda(t)
$$

where $\Lambda(t)$ is a normalized periodic triangular waveform function.

Fig. 13 compares the measured detection results obtained using various upper displacement limits $\left(x_{b \max }\right)$ under the setup with only one radar and the setup with the two-radar array. This setup was made by turning off or on the SIL radar 2 . The one-radar detection results, plotted as solid lines in Fig. 13, indicate that the detected motion signal exhibits low distortion at small $x_{b \max }$ values of, say, less than $1 \mathrm{~cm}$, but becomes obviously distorted as $x_{b \text { max }}$ increases to close to a quarter of a wavelength (around $3.1 \mathrm{~cm}$ ). This result can be explained by the following derivation of the motion signal detected using only one radar. The baseband signal in the SIL radar $1 S_{b b 1}(t)$ is proportional to the frequency change in a dynamic steady state. Assuming no clutter, $S_{b b 1}(t)$ can be derived from (10) as

$$
\begin{aligned}
S_{b b 1}(t) & \propto \Delta \omega(t)=\omega_{\mathrm{LR} 1, d} \sin \phi_{d 1}(t) \\
& =\omega_{\mathrm{LR} 1, d} \sin \left(\frac{2 \omega_{\mathrm{out}}}{c}\left(d_{0}+x_{b}(t)\right)\right)
\end{aligned}
$$

where $c$ is the speed of light. Formula (31) reveals that the distortion of the waveforms in the bottom two graphs of Fig. 13 for the one-radar results arises mainly from the sine term in (31).

As indicated by the dashed lines in Fig. 13, the two-radar array achieves excellent Doppler shift cancellation. To demonstrate the effectiveness of the cancellation, the rms values for the motion signals that are detected by only one radar and by the two-radar array are computed for comparison. The rms values that correspond to the four motion signals with $x_{b \max }$ equal to $1,2,3$, and $4 \mathrm{~cm}$ are $0.702,1.251,1.525$, and $1.546 \mathrm{~V}$, respectively, when one radar is used, but only 0.022, 0.044, 0.087, and $0.092 \mathrm{~V}$, respectively, when the two-radar array is used. The reduction percentages are therefore $96.9 \%, 96.5 \%, 94.3 \%$, and

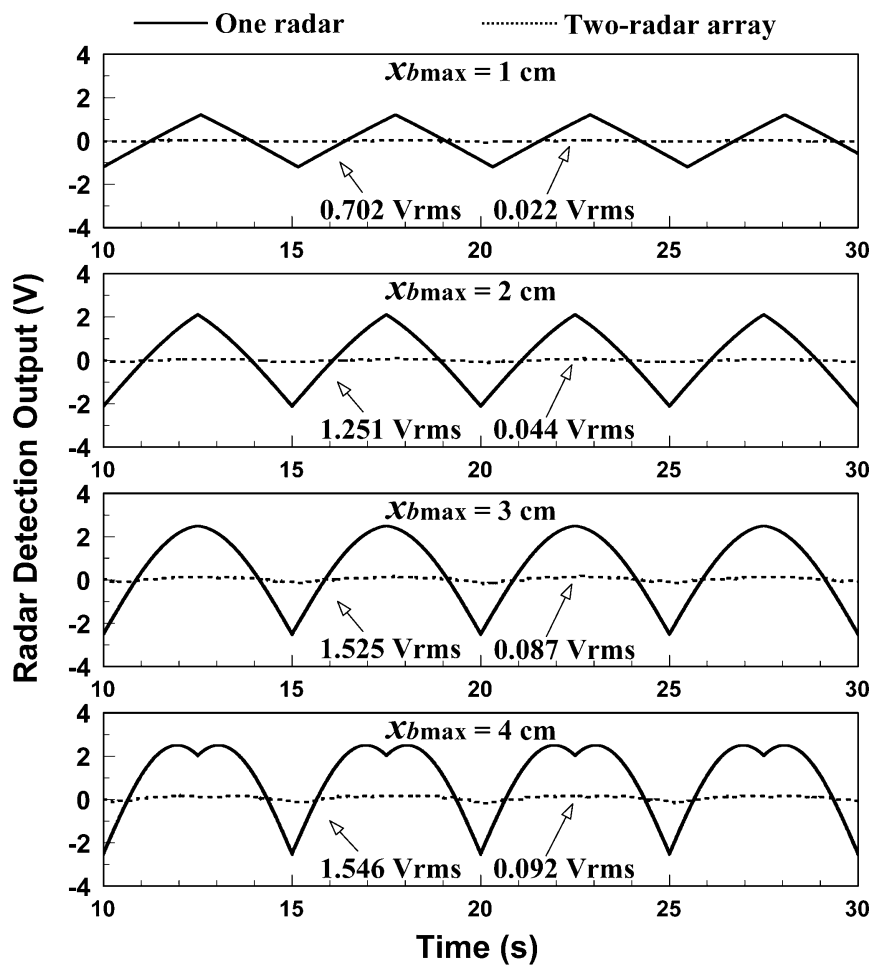

Fig. 13. Detected motion waveforms for the experimental setup in Fig. 12.

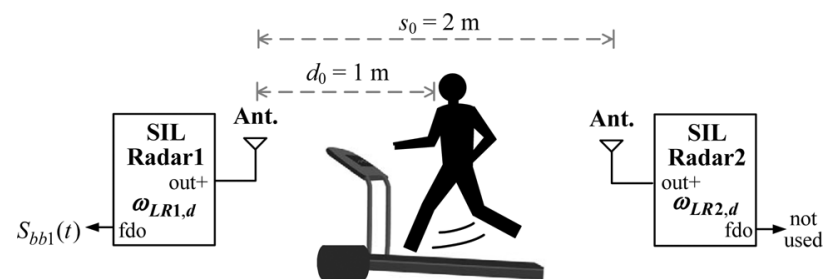

Fig. 14. Experimental setup for using SIL radars to detect vital signs of a subject jogging on a treadmill.

$94.0 \%$, respectively, revealing a decrease in cancellation as the upper displacement limit increases.

Fig. 14 shows the cardiopulmonary monitoring setup for an adult subject who is jogging on a treadmill, which is placed in the middle of the 2-m spaced two-radar array under the same operating conditions as in the preceding experiment. During the sensing period, the subject's movement is restricted to within $10 \mathrm{~cm}$. The subject jogged for over 10 min with random body motion. Fig. 15(a) and (b) plots the detected vital-sign waveforms using a one-radar array and a two-radar array, respectively. Comparing Fig. 15(a) and (b) demonstrates that the tworadar array, with random body movement cancellation, yielded a more uniform waveform than the single radar, whose results include no cancellation, because, in the latter case, random body movement causes a nonuniform vibration in the waveform, but these effects are cancelled in the former case. The Fourier transform of the results in Fig. 16(a) and (b) yields the detection output spectra in Fig. 15(a) and (b). The respiration signal can be clearly identified in the one-radar results, but distinguishing between the heartbeat signal and the random body motion signal is difficult. In contrast, both respiration and heartbeat signals, indicating a breathing rate of 30 breaths/min and a heartbeat rate 


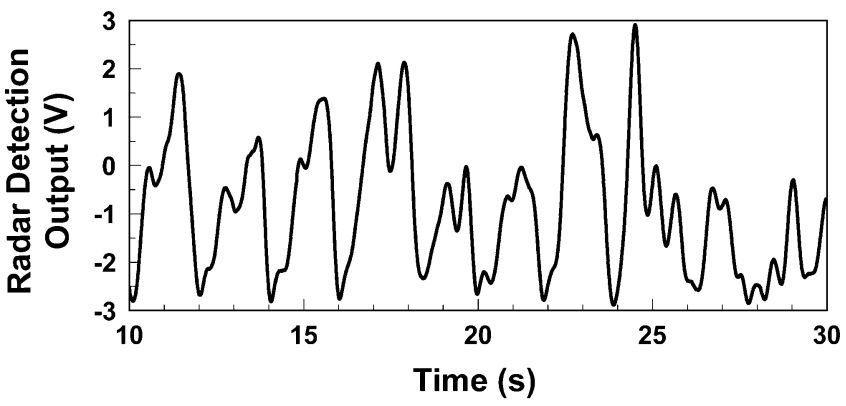

(a)

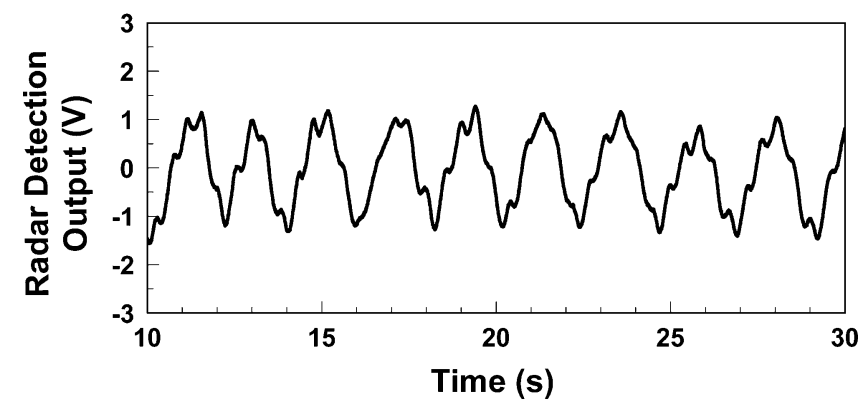

(b)

Fig. 15. Comparison of detected vital-sign waveforms between one SIL radar and two mutually injection-locked SIL radars for the experiment in Fig. 14. (a) One SIL radar. (b) Two mutually injection-locked SIL radars.

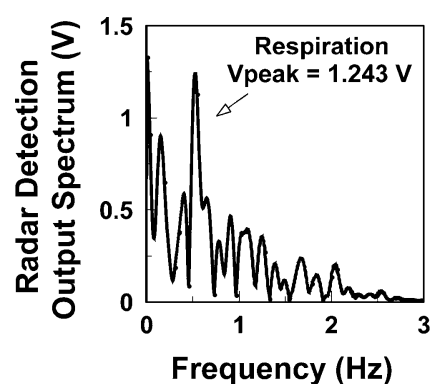

(a)

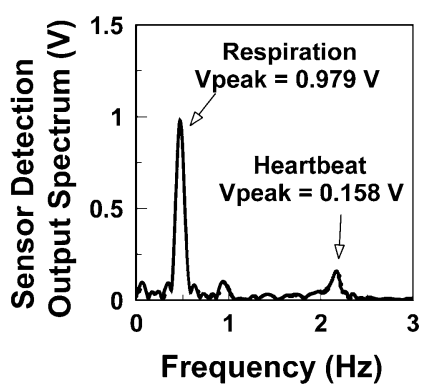

(b)
Fig. 16. Comparison of detected vital-sign spectra between one SIL radar and two mutually injection-locked SIL radars for the experiment in Fig. 14. (a) One SIL radar. (b) Two mutually injection-locked SIL radars.

of 130 beats/min during fitness exercise, can be clearly identified in the two-radar array results with random body movement cancellation.

With the (31), the subject's instantaneous displacement $x_{b}(t)$ can be computed from the data in Fig. 15(a), yielding a result that includes cardiopulmonary motion and random body motion. As shown in Fig. 17, the nonperiodic random body motion can be estimated approximately by further filtering out the breathing contribution.

Attention should be paid to the fact that the MIL signal may be blocked by the subject. Fig. 18 shows the path loss between two antennas of radar array as the subject's position varies. The solid line and dotted line show the measured path losses when the subject is at the center position and at an offset position of $10 \mathrm{~cm}$ from the center, respectively. The difference in path

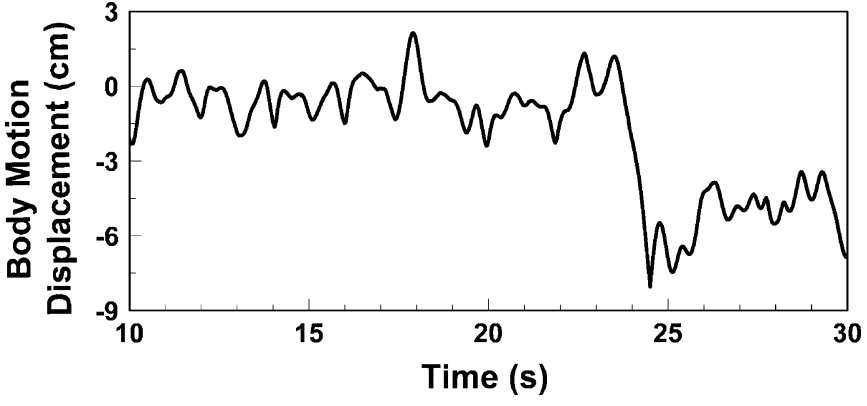

Fig. 17. Random body motion waveform estimated from the radar detection results in Fig. 15(a).

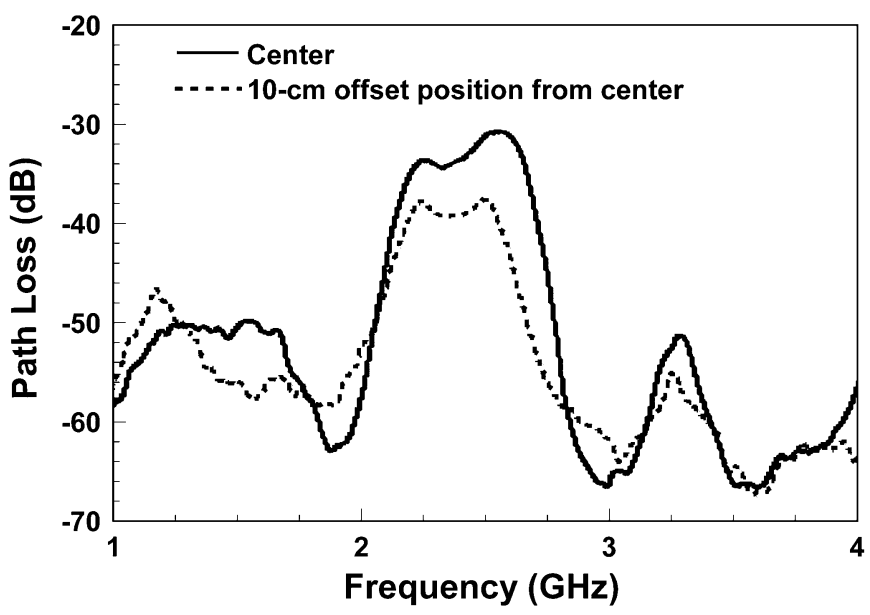

Fig. 18. Measured path loss between two mutually injection-locked SIL radars.

loss at $2.4 \mathrm{GHz}$ is about $5.5 \mathrm{~dB}$. A larger path loss corresponds to a greater difficulty in establishing an effective MIL path for random body movement cancellation. To cancel the effect of larger random body movement, an additional bidirectional amplifier between the antenna and the VCO can be used to increase the locking range of individual single-antenna SIL radars.

\section{CONCLUSION}

This paper presents a single-antenna SIL radar array that can eliminate the effects of random body movement on the detection of vital signs using MIL techniques. First, this work provides a theoretical basis for predicting the SNR gain performance of an SIL radar with clutter effects. The experiments yield very promising results that are consistent with the theoretical predictions. Then, the study provides a frequency-domain model for deriving the conditions under which the Doppler shift that results from random body motion can be cancelled while maximizing the SNR for the proposed radar architecture. Experiments on actuator-controlled motion experiments verify the theoretical predictions of excellent cancellation of the Doppler shift that is caused by back-and-forth motion. In practical applications, the proposed architecture can be used for the high-quality monitoring of the cardiopulmonary activities of a subject who jogs on a treadmill with a considerable degree of random body motion. 


\section{REFERENCES}

[1] A. D. Droitcour, O. Boric-Lubecke, V. M. Lubecke, J. Lin, and G. T. A. Kovac, "Range correlation and I/Q performance benefits in single-chip silicon Doppler radars for noncontact cardiopulmonary monitoring,", IEEE Trans. Microw. Theory Tech, vol. 52, no. 3, pp. 838-848, Mar. 2004.

[2] Y. Yan, C. Li, and J. Lin, "Effects of I/Q mismatch on measurement of periodic movement using a Doppler radar sensor," in IEEE Radio Wireless Symp. Dig., Jan. 2010, pp. 196-199.

[3] Y. Xiao, J. Lin, O. Boric-Lubecke, and V. M. Lubecke, "Frequency tuning technique for remote detection of heartbeat and respiration using low-power double-sideband transmission in the $K a$-band," IEEE Trans. Microw. Theory Tech., vol. 54, no. 5, pp. 2023-2032, May 2006.

[4] F.-K. Wang, C.-J. Li, C.-H. Hsiao, T.-S. Horng, J. Lin, K.-C. Peng, J.-K. Jau, J.-Y. Li, and C.-C. Chen, "A novel vital-sign sensor based on a self-injection-locked oscillator," IEEE Trans. Microw. Theory Tech., vol. 58, no. 12, pp. 4112-4120, Dec. 2010.

[5] B.-K. Park, O. Boric-Lubecke, and V. M. Lubecke, "Arctangent demodulation with dc offset compensation in quadrature Doppler radar receiver systems," IEEE Trans. Microw. Theory Tech., vol. 55, no. 5, pp. 1073-1079, May 2007.

[6] C. Li and J. Lin, "Random body movement cancellation in Doppler radar vital sign detection," IEEE Trans. Microw. Theory Tech., vol. 56, no. 12, pp. 3143-3152, Dec. 2008.

[7] B. Razavi, "Design considerations for direct-conversion receivers," IEEE Trans. Circuits Syst. II, Analog Digit. Signal Process., vol. 44, no. 6, pp. 428-435, Jun. 1997.

[8] T.-Y. Chin, K.-Y. Lin, S.-F. Chang, and C.-C. Chang, "A fast clutter cancellation method in quadrature Doppler radar for noncontact vital signal detection," in IEEE MTT-S Int. Microw. Symp. Dig., May 2010, pp. 764-767.

[9] B.-K. Park, D. Samardzija, V. M. Lubecke, O. Boric-Lubecke, and T. Sizer, "DC offset reduction in phase diversity heterodyne Doppler radar system," in IEEE AP-S Int. Symp. Dig., Jun. 2007, pp. 3884-3887.

[10] J. K. Kiriazi, O. Boric-Lubecke, and V. M. Lubecke, "Considerations in measuring vital signs cross section with Doppler radar," in IEEE Radio Wireless Symp. Dig., Jan. 2011, pp. 426-429.

[11] I. Mostafanezhad, O. Boric-Lubecke, V. Lubecke, and D. P. Mandic, "Application of empirical mode decomposition in removing fidgeting interference in Doppler radar life signs monitoring devices," in Proc. IEEE Eng. Med. Biolo. Soc. Annu. Int. Conf., Sep. 2009, pp. 340-343.

[12] R. Fletcher and J. Han, "Low-cost differential front-end for Doppler radar vital sign monitoring," in IEEE MTT-S Int. Microw. Symp. Dig., Jun. 2009, pp. 1325-1328.

[13] X. Yu, C. Li, and J. Lin, "Two-dimensional noncontact vital sign detection using Doppler radar approach," in IEEE MTT-S Int. Microw. Symp. Dig., Jun. 2011, pp. 1-4, Session TU1B-1.

[14] K. M. Chen, D. Misra, H. Wang, H. R. Chuang, and E. Postow, "An $\mathrm{X}$-band microwave life-detection system," IEEE Trans. Biomed. Eng., vol. BME-33, no. 7, pp. 697-701, Jul. 1986.

[15] K. M. Chen, Y. Huang, J. Shang, and A. Norman, "Microwave life-detection systems for searching human subjects under earthquake rubble or behind barrier," IEEE Trans. Biomed. Eng., vol. 27, pp. 105-114, Jan. 2000.

[16] B.-K. Park, S. Yamada, and V. M. Lubecke, "Measurement method for imbalance factors in direct-conversion quadrature radar systems," IEEE Microw. Wireless Compon. Lett., vol. 17, no. 5, pp. 403-405, May 2007.

[17] P. Liao and R. A. York, "A new phase-shifterless beam-scanning technique using arrays of coupled oscillators," IEEE Trans. Microw. Theory Tech., vol. 41, no. 10, pp. 1810-1815, Oct. 1993.

[18] R. A. York and T. Itoh, "Injection- and phase-locking techniques for beam control," IEEE Trans. Microw. Theory Tech., vol. 46, no. 11, pp. 1920-1929, Nov. 1998.

[19] S.-H. Yan and T.-H. Chu, "A beam-steering antenna array using injection locked coupled oscillators with self-tuning of oscillator free-running frequencies," IEEE Trans. Antennas Propag., vol. 56, no. 9, pp. 2920-2929, Sep. 2008.

[20] F.-K. Wang, T.-S. Horng, K.-C. Peng, J.-K. Jau, J.-Y. Li, and C.-C. Chen, "Mutual injection-locked SIL sensor array for vital sign detection with random body movement cancellation," in IEEE MTT-S Int. Microw. Symp. Dig., Jun. 2011, pp. 1-4, Session TH2C-4.

[21] R. Adler, "A study of locking phenomena in oscillators," Proc. IRE, vol. 34, no. 6, pp. 351-357, Jun. 1946.

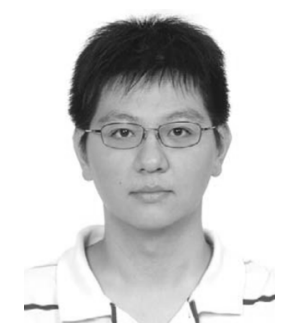

Fu-Kang Wang ( $\left.\mathrm{S}^{\prime} 10\right)$ was born in Kaohsiung, Taiwan, on May 15, 1985. He received the B.S.E.E. and M.S.E.E. degrees from the National Sun Yat-Sen University, Kaohsiung, Taiwan, in 2007 and 2009, respectively, where he is currently working toward the $\mathrm{Ph} . \mathrm{D}$. degree in electrical engineering.

His doctoral work is focused on RF sensing techniques.

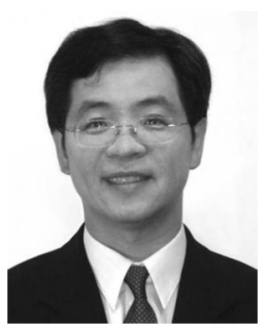

Tzyy-Sheng Horng (S'88-M'92-SM'05) was born in Taichung, Taiwan, on December 7, 1963. He received the B.S.E.E. degree from the National Taiwan University, Taipei, Taiwan, in 1985, and the M.S.E.E. and Ph.D. degrees from the University of California, Los Angeles, in 1990 and 1992, respectively.

Since August 1992, he has been with the Department of Electrical Engineering, National Sun Yat-Sen University, Kaohsiung, Taiwan, where he was the Director of the Telecommunication Research and Development Center (2003-2008) and Director of the Institute of Communications Engineering (2004-2007), and where he is currently a Professor. He has authored or coauthored over 100 technical publications published in refereed journals and conferences proceedings. He holds over ten patents. His research interests include RF and microwave integrated circuits and components, RF signal integrity for wireless system-in-package, and digitally assisted RF technologies.

Dr. Horng has served on several Technical Program Committees of international conferences including the International Association of Science and Technology for Development (IASTED) International Conference on Wireless and Optical Communications, the IEEE Region 10 International Technical Conference, the IEEE International Workshop on Electrical Design of Advanced Packaging and Systems, the AsiaPacific Microwave Conference, the IEEE Radio and Wireless Symposium, and the Electronic Components and Technology Conference. He was the recipient of the 1996 Young Scientist Award presented by the International Union of Radio Science, the 1998 IndustryEducation Cooperation Award presented by the Ministry of Education, Taiwan, and the 2010 Distinguished Electrical Engineer Award presented by the Chinese Institute of Electrical Engineering, Taiwan.

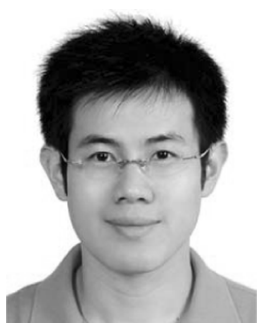

Kang-Chun Peng (S'00-M'05) was born in Taipei, Taiwan, February 18, 1976. He received the B.S.E.E., M.S.E.E., and Ph.D. degrees from the National Sun Yat-Sen University, Kaohsiung, Taiwan, in 1998, 2000 , and 2005, respectively.

$\mathrm{He}$ is currently an Assistant Professor with the Department of Computer and Communication Engineering, National Kaohsiung First University of Science and Technology, Kaohsiung, Taiwan. His current research interests are in the area of delta-sigma modulation techniques, low-noise phase-locked loops, low-power voltage-controlled oscillators, and modulated frequency synthesizers.

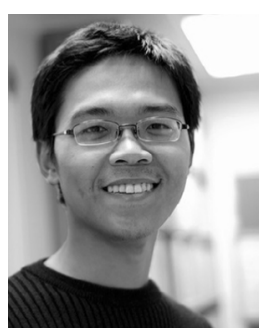

Je-Kuan Jau was born in Tainan, Taiwan, on November 19, 1977. He received the B.S.E.E. and $\mathrm{Ph} . \mathrm{D}$. degrees from National Sun Yat-Sen University, Kaohsiung, Taiwan, in 1999 and 2006, respectively.

$\mathrm{He}$ is currently an Engineerg with the Information and Communications Research Laboratories, Industrial Technology and Research Institute, Hsinchu, Taiwan. His research interests are RF transmitters and power amplifiers. 


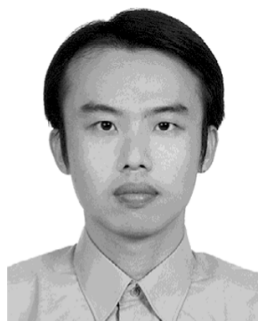

Jian-Yu Li (S'07) was born in Pingtung, Taiwan. $\mathrm{He}$ received the B.S.E.E. and M.S.E.E. degrees from the National Sun Yat-Sen University, Kaohsiung, Taiwan, in 1998 and 2000, respectively. He is currently working toward the Ph.D. degree in communication engineering at National Chiao Tung University, Hsinchu, Taiwan.

In 2000, he joined the Information and Communications Research Laboratories, Industrial Technology and Research Institute, Hsinchu, Taiwan, as an Engineer. His research interests are in the area of microwave integrated circuits and passive components design.

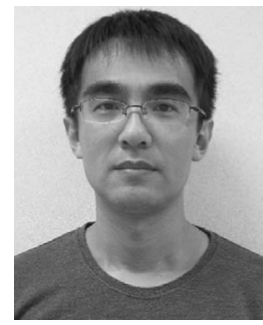

module design.
Cheng-Chung Chen was born in Hsinchu, Taiwan, in 1975. He received the B.S. degree in electrical engineering from the National Sun Yet-Sen University, Kaoshiung, Taiwan, in 1997, and the M.S. and Ph.D. degrees in communication engineering from the National Chiao-Tung University, Hsinchu, Taiwan, in 1999 and 2003, respectively.

In 2003, he joined the Information and Communications Research Laboratory, Industrial Technology and Research Institute, Hsinchu, Taiwan. His research interests include RF front-end chip and 